\title{
Nonequilibrium resonant spectroscopy of molecular vibrons
}

\author{
Dmitry A. Ryndyk and Gianaurelio Cuniberti* \\ Institute for Theoretical Physics, University of Regensburg, D-93040 Regensburg, Germany
}

(Received 8 March 2007; revised manuscript received 21 August 2007; published 24 October 2007)

\begin{abstract}
Quantum transport through single molecules is essentially affected by molecular vibrations. We investigate the behavior of the prototype single-level model with weak to intermediate electron-vibron interactions and arbitrary couplings to the leads. For this, we have developed a nonequilibrium self-consistent theory which allows us to explore the nonperturbative regime via the nonequilibrium Green function formalism. We show that the nonequilibrium resonant spectroscopy is able to determine the energies of molecular orbitals and the spectrum of molecular vibrations. Our results are relevant to scanning tunneling spectroscopy experiments and demonstrate the importance of the systematic and self-consistent investigation of the effects of the vibronic dynamics onto the transport through single molecules.
\end{abstract}

DOI: 10.1103/PhysRevB.76.155430

PACS number(s): 85.65. $+\mathrm{h}, 73.23 .-\mathrm{b}$

\section{INTRODUCTION}

Molecular electronics ${ }^{1-3}$ is one of the most promising developments of nanoelectronics, raising new theoretical challenges and calling for new methodological ideas. The understanding of quantum electron transport at the molecular scale is a key step to future device operation. Recently, the interaction of electrons with molecular vibrations attracted enormous attention unveiled by the successful electron transport experiments with single molecules. ${ }^{4-14}$ In scanning tunneling spectroscopy (STS) experiments, clear signatures of the electron-vibron interaction have been observed. ${ }^{15-18}$ In these experiments, both the electron-vibron interaction and the electron-to-lead coupling are well beyond the perturbation limit, so that a theory beyond linear response or master equation approaches is necessary. In this paper, we present such a theory and discuss the vibronic features in the currentvoltage curves of molecular junctions as a spectroscopic tool.

Resonant electron transport through a molecular orbital with an electron-vibron interaction at finite voltages is essentially different from the well known off-resonant inelastic electron tunneling spectroscopy (IETS). Indeed, in the usual IETS situation, one typically considers multichannel tunneling between two bulk metals with continuous density of states, and an increase of the conductance is observed when the bias voltage exceeds the threshold determined by the frequency of a vibrational mode. As we shall see below, in resonant tunneling through molecular levels coupled to vibrons, the situation is very different: In addition to the usual IETS signal, the inelastic signal can be observed as an extra peak in the differential conductance or just as a decrease of the conductance. The resulting signal is determined by the energy of the electronic state, the vibronic frequency, the electron-vibron coupling, and the molecule-to-lead coupling. The position of the molecular energy levels could be changed by the external gate voltage, but in the STS experiments, the application of the gate voltage is not possible; instead, the tip-to-molecule distance can be changed, also changing the molecule-to-tip coupling. ${ }^{16}$ Thus, it is very important to investigate quantum transport at arbitrary coupling to the leads. The simplest model, which properly describes these peculiarities, is a single-electron level with energy $\epsilon_{0}$ interacting with a single vibron with frequency $\omega_{0}$ (Fig. 1) and coupled to two noninteracting equilibrium electrodes. The dynamics of this minimal model is described in the paper in a self-consistent formalism, which goes beyond perturbation theory.

This paper is organized as follows: After the formulation of the electron-vibron model (Sec. II) and a brief description of the method used (Sec. III), we consider the differential conductance in the simple single-level model and show the effects of vibrons at weak and strong coupling to the leads (Sec. IV).

\section{MODEL}

We analyze here the electron-vibron model ${ }^{19,20}$ by considering a molecule coupled to free conduction electrons in the leads by a usual tunneling Hamiltonian. Furthermore, the electrons are coupled to vibrational modes. The full Hamiltonian is the sum of the molecular Hamiltonian $\hat{H}_{M}$, the Hamiltonians of the leads $\hat{H}_{R(L)}$, the tunneling Hamiltonian $\hat{H}_{T}$ describing the molecule-to-lead coupling, the vibron Hamiltonian $\hat{H}_{V}$ including electron-vibron interaction and coupling of vibrations to the environment (describing dissipation of vibrons)

$$
\hat{H}=\hat{H}_{M}+\hat{H}_{V}+\hat{H}_{L}+\hat{H}_{R}+\hat{H}_{T}
$$

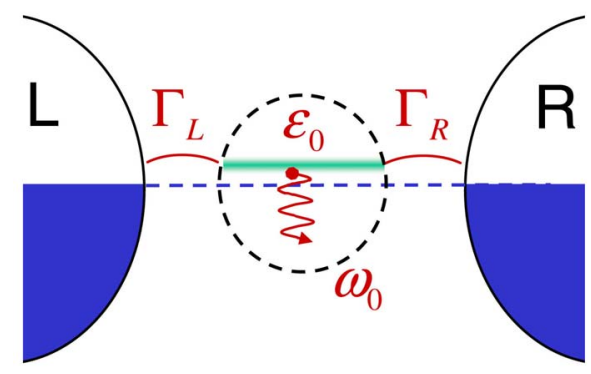

FIG. 1. (Color online) Schematic picture of the considered electron-vibron single-level model, coupled to the left and right leads. 
A molecule is described by a set of localized states $|\alpha\rangle$ with energies $\epsilon_{\alpha}$ and interorbital overlap integrals $t_{\alpha \beta}$ by the following model Hamiltonian:

$$
\hat{H}_{M}^{(0)}=\sum_{\alpha}\left[\epsilon_{\alpha}+e \varphi_{\alpha}(t)\right] d_{\alpha}^{\dagger} d_{\alpha}+\sum_{\alpha \neq \beta} t_{\alpha \beta} d_{\alpha}^{\dagger} d_{\beta},
$$

where $d_{\alpha}^{\dagger}$ and $d_{\alpha}$ are creation and annihilation operators in the states $|\alpha\rangle$ and $\varphi_{\alpha}(t)$ is the (self-consistent) electrical potential. The index $\alpha$ is used to mark single-electron states (atomic orbitals) including the spin degree of freedom. The parameters of a tight-binding model could be determined by ab initio methods. ${ }^{3}$ This is a compromise, which allows us to consider complex molecules with a relatively simple minimal model.

Vibrations and the electron-vibron coupling are described by the Hamiltonian ${ }^{20-23}(\hbar=1)$

$$
\hat{H}_{V}=\sum_{q} \omega_{q} a_{q}^{\dagger} a_{q}+\sum_{\alpha \beta} \sum_{q} \lambda_{\alpha \beta}^{q}\left(a_{q}+a_{q}^{\dagger}\right) d_{\alpha}^{\dagger} d_{\beta} .
$$

Here, vibrations are considered as localized phonons and $q$ is an index labeling them, not the wave vector. The first term describes free vibrons with the energy $\omega_{q}$. The second term represents the electron-vibron interaction. We include both diagonal coupling, which describes a change of the electrostatic energy with the distance between atoms, and the offdiagonal coupling, which describes the dependence of the matrix elements $t_{\alpha \beta}$ over the distance between atoms.

The isolated single-level electron-vibron model is described by the Hamiltonian

$$
\hat{H}_{M+V}=\left(\epsilon_{0}+e \varphi_{0}\right) d^{\dagger} d+\omega_{0} a^{\dagger} a+\lambda\left(a^{\dagger}+a\right) d^{\dagger} d,
$$

where the first and second terms describe the free electron state and the free vibron, and the third term is the electronvibron minimal coupling interaction.

The electrical potential of the molecule $\varphi_{0}$ plays an important role in transport at finite voltages. It describes the shift of the molecular level by the bias voltage, which is divided between the left lead (tip), the right lead (substrate), and the molecule as $\varphi_{0}=\varphi_{R}+\eta\left(\varphi_{L}-\varphi_{R}\right){ }^{24}$ We assume the simplest linear dependence of the molecular potential $(\eta$ $=$ const ), but its nonlinear dependence ${ }^{25}$ can be easily included in our model.

The Hamiltonians of the right $(R)$ and left $(L)$ leads read

$$
\hat{H}_{i=L(R)}=\sum_{k \sigma}\left(\epsilon_{i k \sigma}+e \varphi_{i}\right) c_{i k \sigma}^{\dagger} c_{i k \sigma} .
$$

$\varphi_{i}(t)$ are the electrical potentials of the leads. Finally, the tunneling Hamiltonian

$$
\hat{H}_{T}=\sum_{i=L, R} \sum_{k \sigma, \alpha}\left(V_{i k \sigma, \alpha} c_{i k \sigma}^{\dagger} d_{\alpha}+\text { H.c. }\right)
$$

describes the hopping between the leads and the molecule. A direct hopping between two leads is neglected.

Though the model described above has a long history, the many questions it implies are not answered up to now. While the isolated electron-vibron model can be solved exactly by the so-called polaron or Lang-Firsov transformation, ${ }^{26-28}$ the coupling to the leads produces a true many-body problem.

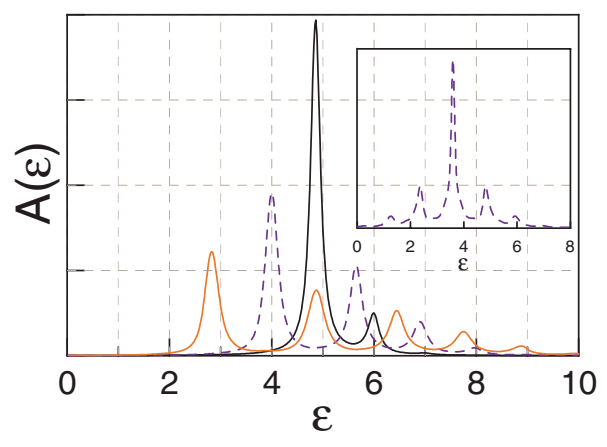

FIG. 2. (Color online) Spectral function at different electronvibron couplings: $\lambda / \omega_{0}=0.4$ (black), $\lambda / \omega_{0}=1.2$ (blue/dark gray, dashed), and $\lambda / \omega_{0}=2$ (red/gray); at $\epsilon_{0} / \omega_{0}=5, \Gamma_{L} / \omega_{0}=\Gamma_{R} / \omega_{0}=0.1$. In the inset, the spectral function at $\lambda / \omega_{0}=1.2$ is shown at a finite voltage when the level is partially filled. Energies are in units of $\hbar \omega_{0}$.

The inelastic resonant tunneling of single electrons through the localized state coupled to phonons was first considered in Refs. 29-32. There, the exact solution in the single-particle approximation was derived, ignoring completely the Fermi sea in the leads. At strong electron-vibron couplings and weak couplings to the leads, the satellites of the main resonant peak are formed in the spectral function (Fig. 2). The number of the essential sidebands is determined by the wellknown Huang-Rhys factor ${ }^{33} S=\left(\lambda / \omega_{0}\right){ }^{2}$ The question which remains is whether these sidebands can be observed in the differential conductance when the coupling to all electrons in the leads should be taken into account simultaneously. New theoretical treatments were presented recently in Refs. 21-23 and 34-51. In parallel, the theory of inelastic resonant tunneling in scanning tunneling spectroscopy was developed. ${ }^{52-57}$ For a recent review of the electron-vibron problem and its relation to the molecular transport, see Ref. 58.

The essential progress in the calculation of transport properties in the strong electron-vibron interaction limit has been made with the help of the master equation approach. . $^{37,40,45-47}$ This method, however, is valid only in the limit of very weak molecule-to-lead coupling and neglects all spectral effects, which are the most important at finite coupling to the leads.

\section{METHOD}

Our approach is based on the nonequilibrium Green function technique, ${ }^{59-61}$ which is now a standard method in mesoscopic physics and molecular electronics. We follow the formulation pioneered by Meir and Wingreen ${ }^{62}$ and Jauho and co-workers. ${ }^{63-65}$ The case of intermediate and strong electron-vibron couplings at finite tunneling rates is the most interesting but also the most difficult. Only the approaches by Flensberg ${ }^{38}$ and Galperin et al. ${ }^{48}$ exist, both starting from the exact solution for the isolated system and then switching on tunneling as a perturbation. Our approach is exact for noninteracting systems with arbitrary tunneling coupling and is approximate in the strong electron-vibron coupling limit without tunneling. Still, its advantage is clear in the truly 
nonequilibrium formulation.

The current in the left $(i=L)$ or right $(i=R)$ contact to the molecule is described by the well-known expression ${ }^{62,63}$

$$
\begin{aligned}
J_{i=L, R}= & \frac{i e}{\hbar} \int \frac{d \boldsymbol{\epsilon}}{2 \pi} \operatorname{Tr}\left\{\boldsymbol { \Gamma } _ { i } ( \epsilon - e \varphi _ { i } ) \left(\mathbf{G}^{<}(\boldsymbol{\epsilon})\right.\right. \\
& \left.\left.+f_{i}^{0}\left(\epsilon-e \varphi_{i}\right)\left[\mathbf{G}^{R}(\epsilon)-\mathbf{G}^{A}(\boldsymbol{\epsilon})\right]\right)\right\},
\end{aligned}
$$

where $f_{i}^{0}(\epsilon)$ is the equilibrium Fermi distribution function with chemical potential $\mu_{i}\left(k_{B}=1\right)$

$$
f_{i}^{0}(\epsilon)=\frac{1}{\exp \left[\left(\epsilon-\mu_{i}\right) / T\right]+1},
$$

and the level-width function $\boldsymbol{\Gamma}_{i=L(R)}(\epsilon) \equiv \Gamma_{i \alpha \beta}(\epsilon)$ is

$$
\Gamma_{i \alpha \beta}(\epsilon)=2 \pi \sum_{k \sigma} V_{i k \sigma, \beta} V_{i k \sigma, \alpha}^{*} \delta\left(\epsilon-\epsilon_{i k \sigma}\right) .
$$

The lesser (retarded, advanced) Green function matrix of a nonequilibrium molecule $\mathbf{G}^{<(R, A)} \equiv G_{\alpha \beta}^{<(R, A)}$ can be found from the Dyson-Keldysh equations in the integral form

$$
\begin{gathered}
\mathbf{G}^{R}(\boldsymbol{\epsilon})=\mathbf{G}_{0}^{R}(\boldsymbol{\epsilon})+\mathbf{G}_{0}^{R}(\boldsymbol{\epsilon}) \boldsymbol{\Sigma}^{R}(\boldsymbol{\epsilon}) \mathbf{G}^{R}(\boldsymbol{\epsilon}), \\
\mathbf{G}^{<}(\boldsymbol{\epsilon})=\mathbf{G}^{R}(\boldsymbol{\epsilon}) \boldsymbol{\Sigma}^{<}(\boldsymbol{\epsilon}) \mathbf{G}^{A}(\boldsymbol{\epsilon}),
\end{gathered}
$$

or from the corresponding equations in the differential form (see, e.g., Refs. 19 and 20 and references therein).

Here,

$$
\mathbf{\Sigma}^{R,<}=\boldsymbol{\Sigma}_{L}^{R,<(T)}+\boldsymbol{\Sigma}_{R}^{R,<(T)}+\boldsymbol{\Sigma}^{R,<(V)}
$$

is the total self-energy of the molecule composed of the tunneling (coupling to the left and right leads) self-energies

$$
\boldsymbol{\Sigma}_{j=L, R}^{R,<(T)} \equiv \Sigma_{j \alpha \beta}^{R,<(T)}=\sum_{k \sigma}\left\{V_{j k \sigma, \alpha}^{*} G_{j k \sigma}^{R,<} V_{j k \sigma, \beta}\right\}
$$

and the vibronic self-energy $\boldsymbol{\Sigma}^{R,<(V)} \equiv \Sigma_{\alpha \beta}^{R,<(V)}$.

For the retarded tunneling self-energy $\Sigma_{i}^{R(T)}$, one obtains

$$
\boldsymbol{\Sigma}_{i}^{R(T)}(\boldsymbol{\epsilon})=\boldsymbol{\Lambda}_{i}\left(\epsilon-e \varphi_{i}\right)-\frac{i}{2} \boldsymbol{\Gamma}_{i}\left(\epsilon-e \varphi_{i}\right)
$$

where $\boldsymbol{\Lambda}_{i}$ is the real part of the self-energy, which can usually be included in the single-particle Hamiltonian $\hat{H}_{M}^{(0)}$, and $\boldsymbol{\Gamma}_{i}$ describes level broadening due to coupling to the leads. For the corresponding lesser function, one finds

$$
\boldsymbol{\Sigma}_{i}^{<(T)}(\boldsymbol{\epsilon})=i \boldsymbol{\Gamma}_{i}\left(\boldsymbol{\epsilon}-e \varphi_{i}\right) f_{i}^{0}\left(\boldsymbol{\epsilon}-e \boldsymbol{\varphi}_{i}\right) .
$$

In the standard self-consistent Born approximation, using the Keldysh technique, one obtains the following for the vibronic self-energies: ${ }^{19-21,23,40-43,54-57,61,64}$

$$
\begin{aligned}
\boldsymbol{\Sigma}^{R(V)}(\boldsymbol{\epsilon})= & \frac{i}{2} \sum_{q} \int \frac{d \omega}{2 \pi}\left(\mathbf{M}^{q} \mathbf{G}_{\epsilon-\omega}^{R} \mathbf{M}^{q} D_{q \omega}^{K}+\mathbf{M}^{q} \mathbf{G}_{\epsilon-\omega}^{K} \mathbf{M}^{q} D_{q \omega}^{R}\right. \\
& \left.-2 D_{q \omega=0}^{(0) R} \mathbf{M}^{q} \operatorname{Tr}\left[\mathbf{G}_{\omega}^{<} \mathbf{M}^{q}\right]\right),
\end{aligned}
$$

$$
\boldsymbol{\Sigma}^{<(V)}(\boldsymbol{\epsilon})=i \sum_{q} \int \frac{d \omega}{2 \pi} \mathbf{M}^{q} \mathbf{G}_{\epsilon-\omega}^{<} \mathbf{M}^{q} D_{q \omega}^{<},
$$

where $\mathbf{G}^{K}=\mathbf{2} \mathbf{G}^{<}+\mathbf{G}^{R}-\mathbf{G}^{A}$ is the Keldysh Green function and $\mathbf{M}^{q} \equiv M_{\alpha \beta}^{q}$.

Here, we assume that the vibrons are in equilibrium and are not excited by the current, so that the self-consistent Born approximation is a good starting point. The vibron Green function is assumed to be equilibrium with the broadening defined by the external thermal bath (see for details Refs. 20, $21,41,42$, and 58).

\section{RESULTS}

For the single-level model, all equations are significantly simplified. Combining $J_{L}$ and $J_{R}$, the expression for the current can be written for energy independent $\Gamma_{L(R)}$ (wideband limit) as

$$
J=\frac{e}{h} \frac{\Gamma_{L} \Gamma_{R}}{\Gamma_{R}+\Gamma_{L}} \int d \epsilon A(\epsilon)\left[f^{0}\left(\epsilon-e \varphi_{L}\right)-f^{0}\left(\epsilon-e \varphi_{R}\right)\right]
$$

It looks as simple as the Landauer-Büttiker formula, but it is not trivial because the spectral density $A(\epsilon)=-2 \operatorname{Im} G^{R}(\epsilon)$ now depends on the distribution function of the electrons in the fluctuating molecule and hence the applied voltage, $\varphi_{L}$ $=-\varphi_{R}=V / 2 .{ }^{19}$ Indeed, $G^{R}(\epsilon)$ can be found from Eq. (10),

$$
G^{R}(\epsilon)=\frac{1}{\epsilon-\tilde{\epsilon}_{0}-\Sigma^{R(V)}(\epsilon)+i\left(\Gamma_{L}+\Gamma_{R}\right) / 2},
$$

where $\Sigma^{R(V)}(\epsilon)$ is a functional of the electron distribution function inside a molecule. Actually, the lesser function $G^{<}(\epsilon)$ is used in the quantum kinetic formalism as a distribution function. In the single-level case, the usual distribution function can be introduced through the relation

$$
G^{<}(\epsilon)=i A(\epsilon) f(\epsilon) .
$$

Note the essential difference between symmetric $\left(\Gamma_{L}\right.$ $=\Gamma_{R}$ ) and asymmetric junctions. It is clear from the noninteracting solution of the transport problem. Neglecting for a moment the vibron self-energies, we obtain the noninteracting distribution function

$$
f(\epsilon)=\frac{\Gamma_{L} f_{L}^{0}\left(\epsilon-e \varphi_{L}\right)+\Gamma_{R} f_{R}^{0}\left(\epsilon-e \varphi_{R}\right)}{\Gamma_{L}+\Gamma_{R}} .
$$

For strongly asymmetric junctions (e.g., $\Gamma_{L} \ll \Gamma_{R}$ ), the distribution function remains close to the equilibrium function in the right lead $f_{R}^{0}\left(\epsilon-e \varphi_{R}\right)$, thus essentially simplifying the solution. On the other hand, for symmetric junctions, the distribution function has the double-step form and is very different from the equilibrium one.

A typical example of the spectral function at zero voltage is shown in Fig. 2. At a finite voltage, it should be calculated self-consistently. In the inset, the spectral function of the symmetric junction at a finite voltage is shown. It is changed essentially because the distribution function is changed. 


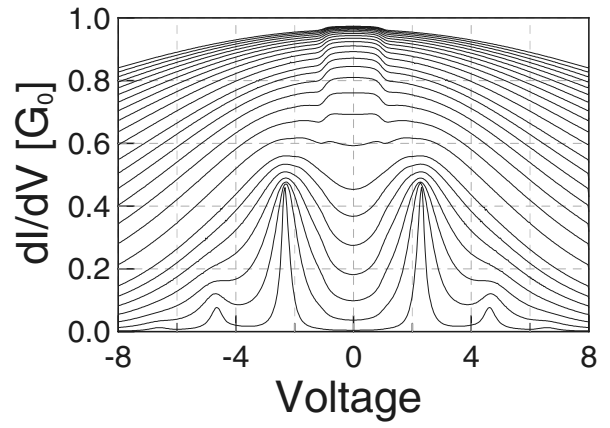

FIG. 3. Differential conductance of a symmetric junction $(\eta$ $\left.=0.5, \Gamma_{R}=\Gamma_{L}\right)$ at different molecule-to-lead couplings, from $\Gamma_{L} / \omega_{0}$ $=0.1$ (lower curve) to $\Gamma_{L} / \omega_{0}=10$ (upper curve), $\lambda / \omega_{0}=1$ and $\epsilon_{0} / \omega_{0}=2$. Voltage is in units of $\hbar \omega_{0} / e$.

Let us discuss a general picture of the vibronic transport in symmetric and asymmetric single-molecule junctions, provided in experiments with the molecular bridges and scanning tunneling microscope (STM)-to-molecule junctions, respectively. The differential conductance, calculated at different molecule-to-lead couplings, is shown in Figs. 3 (symmetric) and 4 (asymmetric). At weak couplings, the vibronic sideband peaks are observed, reproducing the corresponding peaks in the spectral function. At strong couplings, the broadening of the electronic state hides the sidebands, and some features become visible. In the symmetric junction, a suppression of the conductance at $V \simeq \pm \hbar \omega_{0}$ takes place as a result of inelastic scattering of the coherently transformed from the left lead to the right lead electrons. In the asymmetric junction (Fig. 4), the usual IETS increase of the conductance is observed at a negative voltage $V \simeq-\hbar \omega_{0}$; this feature is weak and can be observed only in the incoherent tail of the resonant conductance. We conclude that the vibronic contribution to the conductance can be distinguished clearly in both coherent and tunneling limits.

Now, let us discuss the particular situation of STS experiments. ${ }^{15-18}$ Here, we concentrate mainly on the dependence on the tip-to-molecule distance. ${ }^{16}$ When the tip (left

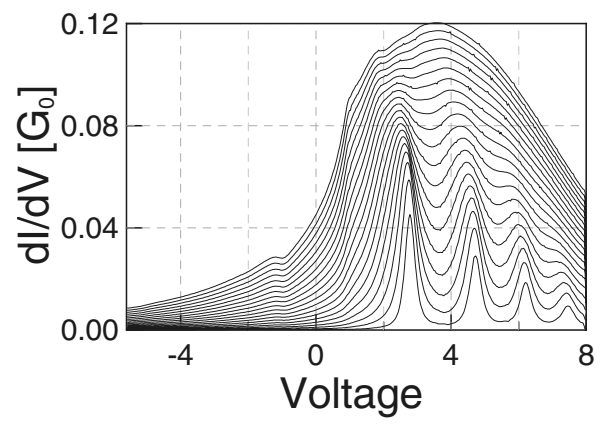

FIG. 4. Differential conductance of an asymmetric junction ( $\eta$ $=0, \Gamma_{R}=20 \Gamma_{L}$ ) at different molecule-to-lead couplings, from $\Gamma_{R} / \omega_{0}=0.2$ (lower curve) to $\Gamma_{R} / \omega_{0}=4$ (upper curve), $\lambda / \omega_{0}=2$ and $\epsilon_{0} / \omega_{0}=5$. The voltage is in units of $\hbar \omega_{0} / e$.

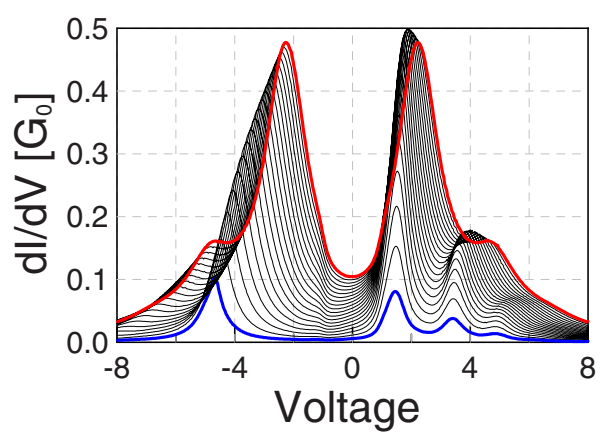

FIG. 5. (Color online) Differential conductance at different molecule-to-STM couplings (see the text) - from asymmetric junction with $\Gamma_{L} / \omega_{0}=0.025, \Gamma_{R} / \omega_{0}=0.5$, and $\eta=0.2$ (lower curve, blue/ dark gray thick line) to symmetric junction with $\Gamma_{L} / \omega_{0}=\Gamma_{R} / \omega_{0}$ $=0.5$, and $\eta=0.5$ (upper curve, red/gray thick line) $-\lambda / \omega_{0}=1$ and $\epsilon_{0} / \omega_{0}=2$. Voltage is in units of $\hbar \omega_{0} / e$.

lead in our notations) is far from the molecule, the junction is strongly asymmetric, $\Gamma_{L} \ll \Gamma_{R}$ and $\eta \rightarrow 0$, and the conductance is similar to that shown in Fig. 4. When the tip is close to the molecule, the junction is approximately symmetric, $\Gamma_{L} \approx \Gamma_{R}$ and $\eta \approx 0.5$, and the conductance curve is of the type shown in Fig. 3. We calculated the transformation of the conductance from the asymmetric to the symmetric case (Fig. 5). It is one feature that appeared in the asymmetric case due to the fact that we started from a finite parameter $\eta=0.2$ (in Fig. 4, $\eta=0$ ), namely, a single peak at negative voltages, which is shifted to a smaller voltage in the symmetric junction. The form and behavior of this peak are in agreement with experimental results. ${ }^{16}$

\section{CONCLUSION}

In conclusion, at weak molecule-to-lead (tip, substrate) coupling, the usual vibronic sideband peaks in the differential conductance are observed; at stronger coupling to the leads (broadening), these peaks are transformed into steplike features. A vibronic-induced decrease of the conductance with voltage is observed in high-conductance junctions. The usual IETS feature (increase of the conductance) can be observed only in the case of low off-resonant conductance. By changing independently the bias voltage and the tip position, it is possible to determine the energy of molecular orbitals and the spectrum of molecular vibrations. In the multilevel systems with a strong electron-electron interaction, further effects, such as Coulomb blockade and Kondo effect, could dominate over the physics which we address here; these effects have to be included in a subsequent step.

\section{ACKNOWLEDGMENTS}

We acknowledge fruitful discussions with Peter Hänggi, Jasha Repp, and Jan van Ruitenbeek. This work was supported by the Volkswagen Foundation Grant No. I/78 340, by the EU under Contract IST-FP6-2001-38951, and by the DFG Grant CU 44/3-2. 
*Present address: Max Bergmann Center for Biomaterials, Dresden University of Technology, D-01062 Dresden, Germany.

${ }^{1}$ M. A. Reed and J. M. Tour, Sci. Am. 282(6), 86 (2000).

${ }^{2}$ C. Joachim, J. K. Gimzewski, and A. Aviram, Nature (London) 408, 541 (2000).

${ }^{3}$ G. Cuniberti, G. Fagas, and K. Richter, Introducing Molecular Electronics (Springer-Verlag, Berlin, 2005).

${ }^{4}$ M. A. Reed, C. Zhou, C. J. Muller, T. P. Burgin, and J. M. Tour, Science 278, 252 (1997).

${ }^{5}$ H. Park, J. Park, A. K. L. Lim, E. H. Anderson, A. P. Alivisatos, and P. L. McEuen, Nature (London) 407, 57 (2000).

${ }^{6}$ J. Park et al., Nature (London) 417, 722 (2002).

${ }^{7}$ W. Liang, M. P. Shores, M. Bockrath, J. R. Long, and H. Park, Nature (London) 417, 725 (2002).

${ }^{8}$ R. H. M. Smit, Y. Noat, C. Untiedt, N. D. Lang, M. C. van Hemert, and J. M. van Ruitenbeek, Nature (London) 419, 906 (2002).

${ }^{9}$ L. H. Yu, Z. K. Keane, J. W. Ciszek, L. Cheng, M. P. Stewart, J. M. Tour, and D. Natelson, Phys. Rev. Lett. 93, 266802 (2004).

${ }^{10}$ L. H. Yu and D. Natelson, Nano Lett. 4, 79 (2004).

${ }^{11}$ M. Elbing, R. Ochs, M. Koentopp, M. Fischer, C. von Hänisch, F. Weigend, F. Evers, H. B. Weber, and M. Mayor, Proc. Natl. Acad. Sci. U.S.A. 102, 8815 (2005).

${ }^{12}$ M. Poot, E. Osorio, K. O’Neill, J. M. Thijssen, D. Vanmaekelbergh, C. A. van Walree, L. W. Jenneskens, and H. S. J. van der Zant, Nano Lett. 6, 1031 (2006).

${ }^{13}$ E. A. Osorio, K. O’Neill, N. Stuhr-Hansen, O. F. Nielsen, T. Bjèrnholm, and H. S. J. van der Zant, Adv. Mater. (Weinheim, Ger.) 19, 281 (2007).

${ }^{14}$ E. Lörtscher, H. B. Weber, and H. Riel, Phys. Rev. Lett. 98, 176807 (2007).

${ }^{15}$ X. H. Qiu, G. V. Nazin, and W. Ho, Phys. Rev. Lett. 92, 206102 (2004).

${ }^{16}$ S. W. Wu, G. V. Nazin, X. Chen, X. H. Qiu, and W. Ho, Phys. Rev. Lett. 93, 236802 (2004).

${ }^{17}$ J. Repp, G. Meyer, S. M. Stojković, A. Gourdon, and C. Joachim, Phys. Rev. Lett. 94, 026803 (2005).

${ }^{18}$ J. Repp, G. Meyer, S. Paavilainen, F. E. Olsson, and M. Persson, Phys. Rev. Lett. 95, 225503 (2005)

${ }^{19}$ D. A. Ryndyk and J. Keller, Phys. Rev. B 71, 073305 (2005).

${ }^{20}$ D. A. Ryndyk, M. Hartung, and G. Cuniberti, Phys. Rev. B 73, 045420 (2006).

${ }^{21}$ T. Frederiksen, M.S. thesis, Technical University of Denmark, 2004.

${ }^{22}$ M. Hartung, M.S. thesis, University of Regensburg, 2004.

${ }^{23}$ T. Frederiksen, M. Brandbyge, N. Lorente, and A.-P. Jauho, Phys. Rev. Lett. 93, 256601 (2004).

${ }^{24}$ S. Datta, W. Tian, S. Hong, R. Reifenberger, J. I. Henderson, and C. P. Kubiak, Phys. Rev. Lett. 79, 2530 (1997).

${ }^{25}$ T. Rakshit, G.-C. Liang, A. W. Ghosh, M. C. Hersam, and S. Datta, Phys. Rev. B 72, 125305 (2005).

${ }^{26}$ I. G. Lang and Y. A. Firsov, Sov. Phys. JETP 16, 1301 (1963).

${ }^{27}$ A. C. Hewson and D. M. Newns, Jpn. J. Appl. Phys., Suppl. 2, 121 (1974).

${ }^{28}$ G. Mahan, Many-Particle Physics, 2nd ed. (Plenum, New York, 1990).

${ }^{29}$ L. I. Glazman and R. I. Shekhter, Zh. Eksp. Teor. Fiz. 94, 292 (1988) [Sov. Phys. JETP 67, 163 (1988)].
${ }^{30}$ N. S. Wingreen, K. W. Jacobsen, and J. W. Wilkins, Phys. Rev. Lett. 61, 1396 (1988).

${ }^{31}$ N. S. Wingreen, K. W. Jacobsen, and J. W. Wilkins, Phys. Rev. B 40, 11834 (1989).

${ }^{32}$ M. Jonson, Phys. Rev. B 39, 5924 (1989).

${ }^{33}$ K. Huang and A. Rhys, Proc. R. Soc. London, Ser. A 204, 406 (1950).

${ }^{34}$ P. Král, Phys. Rev. B 56, 7293 (1997).

${ }^{35}$ U. Lundin and R. H. McKenzie, Phys. Rev. B 66, 075303 (2002).

${ }^{36}$ J.-X. Zhu and A. V. Balatsky, Phys. Rev. B 67, 165326 (2003).

${ }^{37}$ S. Braig and K. Flensberg, Phys. Rev. B 68, 205324 (2003).

${ }^{38}$ K. Flensberg, Phys. Rev. B 68, 205323 (2003).

${ }^{39}$ V. Aji, J. E. Moore, and C. M. Varma, arXiv:cond-mat/0302222 (unpublished).

${ }^{40}$ A. Mitra, I. Aleiner, and A. J. Millis, Phys. Rev. B 69, 245302 (2004).

${ }^{41}$ M. Galperin, M. A. Ratner, and A. Nitzan, Nano Lett. 4, 1605 (2004).

${ }^{42}$ M. Galperin, M. A. Ratner, and A. Nitzan, J. Phys. Chem. 121, 11965 (2004).

${ }^{43}$ M. Galperin, M. A. Ratner, and A. Nitzan, Nano Lett. 5, 125 (2005).

${ }^{44}$ M. Čízěk, M. Thoss, and W. Domcke, Phys. Rev. B 70, 125406 (2004).

${ }^{45}$ J. Koch and F. von Oppen, Phys. Rev. Lett. 94, 206804 (2005).

${ }^{46}$ J. Koch, M. Semmelhack, F. von Oppen, and A. Nitzan, Phys. Rev. B 73, 155306 (2006).

${ }^{47}$ J. Koch, F. von Oppen, and A. V. Andreev, Phys. Rev. B 74, 205438 (2006).

${ }^{48}$ M. Galperin, A. Nitzan, and M. A. Ratner, Phys. Rev. B 73, 045314 (2006).

${ }^{49}$ P. I. Arseyev and N. S. Maslova, JETP Lett. 82, 297 (2005).

${ }^{50}$ P. I. Arseyev and N. S. Maslova, JETP Lett. 84, 93 (2006).

${ }^{51}$ R. Gutiérrez, S. Mohapatra, H. Cohen, D. Porath, and G. Cuniberti, Phys. Rev. B 74, 235105 (2006).

${ }^{52}$ B. N. J. Persson and A. Baratoff, Phys. Rev. Lett. 59, 339 (1987).

${ }^{53}$ M. A. Gata and P. R. Antoniewicz, Phys. Rev. B 47, 13797 (1993).

${ }^{54}$ S. Tikhodeev, M. Natario, K. Makoshi, T. Mii, and H. Ueba, Surf. Sci. 493, 63 (2001).

${ }^{55}$ T. Mii, S. Tikhodeev, and H. Ueba, Surf. Sci. 502, 26 (2002).

${ }^{56}$ T. Mii, S. G. Tikhodeev, and H. Ueba, Phys. Rev. B 68, 205406 (2003).

${ }^{57}$ S. G. Tikhodeev and H. Ueba, Phys. Rev. B 70, 125414 (2004).

${ }^{58}$ M. Galperin, M. A. Ratner, and A. Nitzan, J. Phys.: Condens. Matter 19, 103201 (2007).

${ }^{59}$ L. Kadanoff and G. Baym, Quantum Statistical Mechanics (Benjamin, New York, 1962).

${ }^{60}$ L. V. Keldysh, Zh. Eksp. Teor. Fiz. 47, 1515 (1964) [Sov. Phys. JETP 20, 1018 (1965)].

${ }^{61}$ J. Rammer and H. Smith, Rev. Mod. Phys. 58, 323 (1986).

${ }^{62}$ Y. Meir and N. S. Wingreen, Phys. Rev. Lett. 68, 2512 (1992).

${ }^{63}$ A.-P. Jauho, N. S. Wingreen, and Y. Meir, Phys. Rev. B 50, 5528 (1994).

${ }^{64}$ H. Haug and A.-P. Jauho, Quantum Kinetics and Optics of Semiconductors, Springer Series in Solid-State Sciences Vol. 123 (Springer, New York, 1996).

${ }^{65}$ A.-P. Jauho, J. Phys.: Conf. Ser. 35, 313 (2006). 